\title{
COST-BENEFIT ANALYSIS OF HACCP IMPLEMENTATION IN THE CHINESE SLAUGHTERING AND MEAT PRODUCT PROCESSING INDUSTRY
}

\author{
Shunlong Gong ${ }^{1}$, Chenglin $\mathrm{Ma}^{2}$, Yinsheng Yang ${ }^{2}, \mathrm{Li} \mathrm{Bai}^{2,{ }^{*}}$, Linyi $\mathrm{He}^{3}$ \\ ${ }^{1}$ College of Management, Jilin University, 5988 Renmin Street, Changchun 130025, PR \\ China \\ ${ }^{2}$ College of Biological and Agricultural Engineering, Jilin University, 5988 Renmin Street, \\ Changchun 130025, PR China \\ ${ }^{3}$ College of Management, Beijing Normal University, Beijing 100875, PR China \\ * Corresponding author: College of Biological and Agricultural Engineering, Jilin University, \\ 5988 Renmin Street, Changchun 130025, PR China, Tel: +86 13504334103; E-mail: \\ bl@jlu.edu.cn (L.Bai)
}

\begin{abstract}
The paper reports the results of a study of the costs and benefits associated with the implementation and operation of HACCP in the Chinese slaughtering and meat product processing industry. The research results suggest that although some kinds of intangible costs are more regularly referred to, the major costs of implementing and operating HACCP in the industry are still relatively tangible, such as investment in new equipments and product testing. And although most respondents indicated that the costs of implementing and operating HACCP were approximately in accordance with their prior expectations, still a significant majority indicated that some costs exceeded their expectations. The results also suggest that the slaughtering and meat product processing enterprises do derive benefits from implementing and operating HACCP, and some of them have derived distinct benefits. The results have implications for the further adoption of HACCP not only in the industry itself but also in the Chinese food industry as a whole. Policy makers should take account of these research results and make more quantitative researches to offer more comprehensive and classified information to help food enterprises make decisions on HACCP implementation and operation.
\end{abstract}

Keywords: cost-benefit analysis; HACCP; slaughtering and meat product processing industry

Please use the following format when citing this chapter:

Gong, S., Ma, C., Yang, Y., Bai, L. and He, L., 2009, in IFIP International Federation for Information Processing, Volume 295, Computer and Computing Technologies in Agriculture II, Volume 3, eds. D. Li, Z. Chunjiang, (Boston: Springer), pp. 1681-1689. 


\section{INTRODUCTION}

Hazard Analysis and Critical Control Point (HACCP) system was designed to focus on prevention of possible hazards and to improve the process (Swanson and Anderson), and has been developed and promoted in many countries to obtain better food safety. Although HACCP do help enterprises improve food quality (Gillespie et al., 2000) and may support inspection of regulatory authorities (WHO, 1993), the implementation of the HACCP system requires additional resources for staff training, investments in buildings and equipments, extra purchase of supplies as well as technical support (Henson, Holt, \& Northen, 1999). Like other kinds of enterprises, food enterprises are in pursuit of profits as well. Therefore, inadequate information on costs and benefits have prevented food enterprises from implementing $H A C C P$ to a certain extent since it is important for them to possess necessary information to concretely evaluate the economic impacts prior to implementation.

And the published studies showed that the costs and benefits for food enterprises to implement and operate $H A C C P$ varied from country to country. In the companies of Northern Greece, the major costs of implementing and operating $H A C C P$ were respectively staff training and product testing, and the benefits were ascribed to 3 headings: clientele benefits, product improvements and improvements in production procedures (Semos and Kontogeorgos, 2007). And in the UK dairy processing plants, the major cost of implementing and operating $H A C C P$ was staff time required to document the system, and the most important benefit was the enhanced ability to retain existing customers (Henson, Holt \& Northen, 1999). However, in the Mexican meat plants, investment in new equipment and microbiological tests of products accounted for most of the implementation and operational costs, and the main benefit is reduction in microbial counts (Maldonado et al., 2005).

However, there is little published information on the status of adoption of $H A C C P$ system in the food industry of China, and the resulted costs and benefits that come with the system. Bai L. (2005), et al. depicted the features of the China's food enterprises which have implemented the HACCP system, which laid a foundation for the further researches in the field. Liu et al (2006). reported that the large-sized food enterprises in China knows HACCP system well and have implemented either ISO, GMP, SSOP or $H A C C P$ system; however, the small-sized enterprises in China know $H A C C P$ system little and most of them lack enough motivation to implement the HACCP. Although Wang Z.G., et al (2006). verified the effectiveness of $H A C C P$ on the firms' economic performance, their verification is based on the financial data in the first year of $H A C C P$ implementation rather than the real costs and benefits coming from the implementation of the system. 
Moreover, Wang et al. didn't identify the major costs and benefits of the implementation of $H A C C P$ in the food industry.

The aim of this paper is to explore the process of $H A C C P$ implementation in the food industry of China. The paper reports the initial results of a survey that addresses the following questions in turn: What are the major costs of implementing and operating $H A C C P$ ? What are the major benefits?

\section{MATERIALS AND METHODS}

In September, with the help of Jilin Food and Drug Administration in Jilin Province, northeast of China, questionnaires were sent by faxes and emails to the forty-nine slaughtering and meat product processing enterprises who wish to be involved. Referring to previous researches in this field, the questionnaire was comprised of three sections. The first section investigated the status of the quality assurance systems, the second examined the costs and benefits derived by the $H A C C P$ implementation and operation, and the last one dealt with the general data of the surveyed enterprises.

The structure of the questionnaire and the list of costs and benefits referred to several researchers in the specific field of cost-benefit analysis of HACCP or ISO implementation and operation (see for example Bredahl and Holleran, 1997; Henson, Holt and Northen, 1999; Maldonado et al., 2005). And personal interviews, based on a pilot questionnaire, were used to form the final questionnaire. Personal interviews were conducted in three food companies located in the city of Changchun (Northeast China).

The final form of the questionnaire was sent, with the help of Jilin Food and Drug Administration in Jilin Province, to the forty-nine slaughtering and meat product processing enterprises in September. And a reminder was mailed to non-respondents at the end of October, 2007. Finally, a total of twenty-six questionnaires were returned, and only twenty-one of them, locating in eighteen provinces in China, were fully completed and accepted for survey purposes. Some enterprises refused to return questionnaires for the following reasons of "confidential information", "incapable of answering", "persons concerned unavailable" or "no responsibility for offering information to scientific institution", etc.

Although only twenty-one questionnaires were available, it was considered sufficient enough to serve the objectives of such a survey for three reasons. Firstly, the top 3 of the slaughtering and meat product processing enterprises, Shineway (Shuang Hui), Yurun (Yu Run) and Jinluo (Jin Luo), were involved in the research, and their sales revenue occupied more than 10 percent of the total revenue of all the Chinese slaughtering and meat product processing enterprises in 2006. Secondly, the sales revenue of 
the 21 respondents almost occupied one fourth of the total revenue of the industry. Thirdly, the paper is still a tentative descriptive analysis aiming at inspiring further exploration and quantitative investigation in the field.

The Statistical Package for the Social Sciences - SPSS (Release 12.0, September 2003) for Windows was used to analyze the data. Descriptive statistics was used to describe the HACCP implementation status and the general data of the respondents.

\section{RESULTS AND DISCUSSION}

According to the Interim Measures for Statistical Definitions of Large, Medium and Small Enterprises (the State Economic and Trade Commission, No. 143, 2003) promulgated by the State Economic and Trade Commission, the State Development Planning Commission, the Ministry of Finance and the National Bureau of Statistics, eight (38.1\%) of the twenty-one surveyed respondents were large-sized (employees over 2000, sales over $R M B 300$ million Yuan and total assets over $R M B 400$ million Yuan), ten (47.6\%) were medium-sized (employees ranged from 300 to 2000, sales ranged from $R M B 30$ million to 300 million Yuan and total assets ranged from $R M B 40$ million to 400 million Yuan) and $14.3 \%$ were small-sized (employees less than 300, sales less than $R M B 30$ million Yuan and total assets less than $R M B 40$ million Yuan). Considering the higher proportion of small-sized food enterprises in China, the results showed that more large-sized and medium-sized food enterprises implemented the HACCP system than smallsized ones did.

The product types of the survey respondents are frozen meat, cooked meat and iced fresh meat. And more than one thirds of the respondents also deal in the fields of livestock and poultry breeding, Biological drugs and concentrated feedstuff. Only five of the respondents just distribute products in domestic market. And the top five exporting countries of the sixteen respondents are Hong Kong, Russia, Singapore, Japan and Korea.

$100 \%$ of the respondents claimed that they had full operation of $H A C C P$ system in place. And eighteen $(85.7 \%)$ of them had a fully operational HACCP system for more than twelve months, and only one (4.8\%) less than six months. Respondents were asked to indicate how long it took from their starting to implement $H A C C P$ system to fully operating it. Sixteen $(76.2 \%)$ of them indicated that the whole process took 6-12 months. However, four $(19.0 \%)$ indicated that it took more than 18 months. 


\subsection{Costs of implementing and operating HACCP}

Respondents were presented with a list of costs which previous studies have suggested can be incurred when implementing and operating quality control/assurance systems such as HACCP or ISO 9000 (for example Vanguard Consulting, 1993; Bredahl and Holleran, 1997; Maldonado et al., 2005). They were then asked to rank these costs, a score of one meaning major importance and of zero if a cost not incurred. Respondents were allowed to give a score of one to none, one or more items.

\subsubsection{Costs of implementing HACCP}

There was great variation in the costs of implementing $H A C C P$ among individual respondents (Table 1). For example, although $33.3 \%$ of the respondents judged investment in new equipments to be the most important cost associated with the implementation of $H A C C P, 37.7 \%$ of them had not incurred that cost at all. In a whole, more medium-sized and small-sized enterprises took the cost of investment in new equipment as the most important cost, while more large-sized ones took staff training as the most important one. As showed in table 1, staff time in documenting system was the most frequently incurred cost, being ranked zero by none of the respondents, and one by $19.0 \%$ of the respondents.

Table 1. Rank score for the costs of implementing HACCP

\begin{tabular}{lcc}
\hline \multicolumn{1}{c}{ Costs } & $\begin{array}{c}\text { Proportion of respondents } \\
\text { giving rank of zero (\%) }\end{array}$ & $\begin{array}{c}\text { Proportion of respondents } \\
\text { giving rank of one (\%) }\end{array}$ \\
\hline External consultants & 48.3 & 8.3 \\
Investment in new equipments & 37.7 & 33.3 \\
Staff training & 10.0 & 28.6 \\
Managerial changes & 50.0 & 14.2 \\
New employment of Personal & 66.7 & 8.3 \\
Staff time in documenting system & 0.0 & 19.0 \\
\hline
\end{tabular}

Furthermore, sixteen (76.2\%) of the twenty-one respondents had conducted a formal cost analysis before implementing $H A C C P$ in their enterprises. eleven $(66.7 \%)$ of the 16 responded that the actual implementation costs were almost the same with their expectations, while five $(23.8 \%)$ underestimated the implementation costs. And among the lists of underestimated costs, the costs of staff training and investment in new equipments, which was reported by medium-sized and small-sized enterprises, were referred to more regularly. 


\subsubsection{Costs of operating HACCP}

The respondents were asked to rank the individual costs of operating $H A C C P$ in a manner similar to that described above. Like the costs of implementing $H A C C P$, there was still great variation among the respondents (Table 2). Considering the results as a whole, record keeping was the most frequently incurred cost (ranked zero by none of the respondents), but product testing was ranked as the most important cost (ranked one by $41.7 \%$ of the respondents).

Table 2. Rank scores for the costs of operating HACCP

\begin{tabular}{lcc}
\hline \multirow{2}{*}{ Costs } & $\begin{array}{c}\text { Proportion of respondents } \\
\text { giving rank of zero (\%) }\end{array}$ & $\begin{array}{c}\text { Proportion of respondents } \\
\text { giving rank of one (\%) }\end{array}$ \\
\hline ecord keeping & 0.0 & 23.8 \\
roduct testing & 19.0 & 41.7 \\
taff training & 28.6 & 19.0 \\
lanagerial/supervisory time & 52.4 & 14.3 \\
\hline
\end{tabular}

Seven $(33.3 \%)$ of the respondents indicated that they underestimated the operating costs and the most widely underestimated cost were product testing and record keeping, while the others indicated that the actual operating costs were almost same with their expectation. And twelve (52.4\%) of the respondents indicated that their total production cost had increased after implementing and operating the system. Three (14.3\%) indicated that their total production cost decreased after implementing and operating the system. And after implementing and operating $H A C C P$ system, more smallsized respondents indicated an increase in total production cost than middlesized and large-sized respondents did. $64.3 \%$ of the respondents with employees less than 1000 indicated an increase in total production cost, while only $37.6 \%$ of the respondents with employees over 1000 indicated an increase. Although the costs of implementing and operating HACCP varied greatly among the respondents, the most regularly occurred and important costs were relatively tangible costs, like the cost of product testing, rather than the intangible costs as reported by some other researchers.

However, as mentioned by many researchers (for example Maldonado et al., 2005), the reliability of these results on cost analysis depends on whether the respondents could clearly identify and distinguish the costs of implementing and operating $H A C C P$.

\subsection{Benefits of implementing and operating HACCP}

Respondents were presented with a list of benefits which referred to previous studies and were adapted to the Chinese food enterprises based on a pilot survey in three enterprises. The respondents were asked to rank these 
benefits, a score of one meaning major importance and of zero if a cost not incurred. Respondents were allowed to give a score of one to none, one or more items.

A relatively higher proportion of the respondents allocated zero ranks to each of the possible benefits, indicating significant differences in the types of benefits derived from implementing and operating $H A C C P$ among different enterprises. As showed in table 3, according to the one scores allocated by the respondents, the top three benefits were increased ability to retain existing customers, reduced product microbial counts and increased ability to access new overseas market.

Table 3 Rank scores for benefits of implementing and operating $H A C C P$

\begin{tabular}{lcc}
\hline \multicolumn{1}{c}{ Benefits } & $\begin{array}{c}\text { Proportion of respondents } \\
\text { giving rank of zero (\%) }\end{array}$ & $\begin{array}{c}\text { Proportion of respondents } \\
\text { giving rank of one (\%) }\end{array}$ \\
\hline icreased ability to improve production procedures & $38.1 \%$ & $14.3 \%$ \\
nproved staffs' consciousness of food safety & $47.6 \%$ & $9.5 \%$ \\
icreased product sales & $47.6 \%$ & $9.5 \%$ \\
icreased ability to access new overseas market & $42.9 \%$ & $19.0 \%$ \\
icreased ability to attract new customers & $38.1 \%$ & $14.3 \%$ \\
icreased ability to retain existing customers & $33.3 \%$ & $23.8 \%$ \\
educed product microbial counts & $33.3 \%$ & $19.0 \%$ \\
educed product wastage & $52.4 \%$ & $9.5 \%$ \\
creased product prices & $61.9 \%$ & $4.8 \%$ \\
icreased product shelf life & $75.0 \%$ & $9.5 \%$ \\
creased motivation of supervisory/managerial staff & $80.9 \%$ & $0.0 \%$ \\
\hline
\end{tabular}

Results also showed that all of the respondents indicated that they did derive benefits and nine of them (42.9\%) indicated having derived distinct benefits. And the majority (76.2\%) of the survey respondents indicated that the benefits they had experienced from implementing and operating $H A C C P$ were approximately in accordance with their prior expectations, and 28.6\% of the survey respondents indicated that some intangible benefits, such as increased ability to retain existing customers and increased ability to access new overseas market were even higher than their expectation. However, a significant minority $(23.8 \%)$, especially small-sized enterprises, indicated that some benefits, like increased product prices and increased sales were lower than they had expected.

However, as mentioned by many researchers, these results on benefits presented above should be interpreted with some caution. Given the intangible nature of many of the potential benefits of $H A C C P$ and the fact that a higher proportion of Chinese food enterprises do not formally monitor the costs and benefits of $H A C C P$, many respondents may not have been aware of the benefits and their degree that $H A C C P$ had on their operations. 


\section{CONCLUSIONS AND SUGGESTIONS}

\subsection{Conclusions}

The research results on the costs of implementing and operating $H A C C P$ showed that although most slaughtering and meat product processing enterprises indicated that the costs they had experienced from implementing and operating $H A C C P$ were approximately in accordance with their prior expectations, still a significant proportion of the survey respondents indicated some costs exceeded their expectations.

And the research results on the benefits of implementing and operating $H A C C P$ showed that: firstly, slaughtering and meat product processing enterprises can derive benefits from implementing $H A C C P$ system and some of them have derived distinct benefits; secondly, the benefits from implementing the system are relatively intangible; thirdly, still a significant minority, especially small-sized enterprises, indicates that some benefits, like increased product prices and increased sales are lower than their expectation.

\subsection{Suggestions}

Firstly, policy makers should take account of these research results and make more quantitative researches on the costs and benefits of implementing and operating $H A C C P$ to offer more comprehensive and classified information to help food enterprises make decisions on HACCP implementation and operation.

Secondly, policy makers should also take due regard of these costs and benefits when making $H A C C P$ relative regulation policies or offering directions and destructions to support food enterprises to implement the system.

Thirdly, policy makers can effectively support food enterprises by providing interest subsidy to purchase necessary quality control facilities and offering free manpower training since the major costs of implementing and operating $H A C C P$ in slaughtering and meat product processing enterprises were still relatively tangible, such as investment in new equipments and product testing.

Fourthly, the research results also suggest that policy-makers should take due regard of the differences of costs and benefits among different sized enterprises when formulating regulation and/or support as part of measures designed to promote $H A C C P$ adoption since it seems that the costs of implementing and operating $H A C C P$ of small-sized enterprises are different from those of large-sized ones. 


\section{ACKNOWLEDGEMENTS}

The research is sponsored by Humanities and Social Science Project of Ministry of Education of China (06JC630013). The opinions expressed here do not necessarily reflect those of the funding agency. We also thank the Editor and the anonymous referees for helpful comments and suggestions.

\section{REFERENCES}

Bai, L., Ma, C. L., Gong, S. L., \& Yang, Y. S. Implementation of HACCP system in China: A survey of food enterprises involved. Food Control, 2007, 18: 1108 - 1112.

Bredahl, M., Holleran, E. Transaction costs and institutional innovation in the British food sector. Food Safety, 1997, 3: 403 - 419.

Gillespie, I., Little, C. and Mitchell, R. "Microbiological examination of cold ready-to-eat sliced meats from catering establishments in the United Kingdom", Journal of Applied Microbiology, 2000, 88: 467 - 474.

Henson,S.J., Holt,G. and Northen, J. Cost and benefits of implementing HACCP in the UK dairy processing sector, Food Control, 1999, 10: 99 - 106.

Liu X., Zheng F. T. and Liu, J. H. Industry cognition, implementation and orientation of diffusing HACCP: Demonstrating study on survey samples. Busincess economics and administration, 2006, 15 (10): 39 - 43.

Maldonado E. S., et al. Cost-benefit analysis of HACCP implementation in the Mexican meat industry. Food Control, 2005, 16: 375 - 381.

Seddon, J., Davis, R., Loughran, M., Murrell, R. Implementation and value added: A survey of registered companies. 1993, Vanguard Consulting Ltd, Buckingham.

Semos, A. and Kontogeorgosn, A. HACCP implementation in northern Greece - Food companies' perception of costs and benefits. British Food Journal, 2007, 109 (1): 5 - 19

Swanson, K. M. J. and Anderson, J. E. Industry perspective on the use of microbial data for HACCP validationand verification. Journal of Food Protection, 2000, 63 (6): 815 - 818.

Wang, Z. G., Weng, Y. Z., Yang, Z. G. and Zheng, F. T. The effectiveness of adopting HACCP system certification in food industry: From the questionnaire of 482 food manufacturing enterprises in China. China Soft Science, 2006, 9: 69 - 76.

WHO (1993), Training considerations for the application of the hazard analysis critical control points system to food processing and manufacturing, WHO Document, WHO/FNU/FOS/93.3, World Health Organization, Division of Food and Nutrition, 